OPEN ACCESS

Edited by:

Robert Czajkowski,

University of Gdańsk, Poland

Reviewed by:

Edel Pérez-López,

Laval University, Canada

Christophe Garcion,

INRA Centre Bordeaux-Aquitaine,

France

${ }^{*}$ Correspondence:

Jun-Yi Yang

jyang@nchu.edu.tw

Chih-Horng Kuo

chk@gate.sinica.edu.tw

Specialty section This article was submitted to Microbial Symbioses,

a section of the journal

Frontiers in Microbiology

Received: 10 September 2021

Accepted: 07 February 2022

Published: 28 February 2022

Citation:

Huang C-T, Cho S-T, Lin Y-C,

Tan C-M, Chiu Y-C, Yang J-Y and

Kuo C-H (2022) Comparative Genome Analysis of 'Candidatus

Phytoplasma luffae' Reveals

the Influential Roles of Potential

Mobile Units in Phytoplasma

Evolution

Front. Microbiol. 13:773608. doi: 10.3389/fmicb.2022.773608

\section{Comparative Genome Analysis of 'Candidatus Phytoplasma luffae' Reveals the Influential Roles of Potential Mobile Units in Phytoplasma Evolution}

\author{
Ching-Ting Huang ${ }^{1}$, Shu-Ting Cho', Yu-Chen Lin ${ }^{1}$, Choon-Meng Tan², Yi-Ching Chiu' ${ }^{2,3}$, \\ Jun-Yi Yang ${ }^{2,3,4 *}$ and Chih-Horng Kuo ${ }^{1,3,5,6 *}$
}

${ }^{1}$ Institute of Plant and Microbial Biology, Academia Sinica, Taipei, Taiwan, ${ }^{2}$ Institute of Biochemistry, National Chung Hsing University, Taichung, Taiwan, ${ }^{3}$ Ph.D. Program in Microbial Genomics, National Chung Hsing University and Academia Sinica, Taichung, Taiwan, ${ }^{4}$ Advanced Plant Biotechnology Center, National Chung Hsing University, Taichung, Taiwan, ${ }^{5}$ Molecular and Biological Agricultural Sciences Program, Taiwan International Graduate Program, National Chung-Hsing University and Academia Sinica, Taipei, Taiwan, ${ }^{6}$ Biotechnology Center, National Chung Hsing University, Taichung, Taiwan

Phytoplasmas are insect-transmitted plant pathogens that cause substantial losses in agriculture. In addition to economic impact, phytoplasmas induce distinct disease symptoms in infected plants, thus attracting attention for research on molecular plant-microbe interactions and plant developmental processes. Due to the difficulty of establishing an axenic culture of these bacteria, culture-independent genome characterization is a crucial tool for phytoplasma research. However, phytoplasma genomes have strong nucleotide composition biases and are repetitive, which make it challenging to produce complete assemblies. In this study, we utilized Illumina and Oxford Nanopore sequencing technologies to obtain the complete genome sequence of 'Candidatus Phytoplasma luffae' strain NCHU2019 that is associated with witches' broom disease of loofah (Luffa aegyptiaca) in Taiwan. The fully assembled circular chromosome is $769 \mathrm{~kb}$ in size and is the first representative genome sequence of group 16 SrVIII phytoplasmas. Comparative analysis with other phytoplasmas revealed that NCHU2019 has a remarkably repetitive genome, possessing a pair of $75 \mathrm{~kb}$ repeats and at least 13 potential mobile units (PMUs) that account for 25\% of its chromosome. This level of genome repetitiveness is exceptional for bacteria, particularly among obligate pathogens with reduced genomes. Our genus-level analysis of PMUs demonstrated that these phytoplasma-specific mobile genetic elements can be classified into three major types that differ in gene organization and phylogenetic distribution. Notably, PMU abundance explains nearly $80 \%$ of the variance in phytoplasma genome sizes, a finding that provides a quantitative estimate for the importance of PMUs in phytoplasma genome variability. Finally, our investigation found that in addition to horizontal gene transfer, PMUs also contribute to intra-genomic duplications of effector 
genes, which may provide redundancy for subfunctionalization or neofunctionalization. Taken together, this work improves the taxon sampling for phytoplasma genome research and provides novel information regarding the roles of mobile genetic elements in phytoplasma evolution.

Keywords: plant pathogen, phytoplasma, genomics, molecular evolution, mobile genetic element, effector

\section{INTRODUCTION}

Phytoplasmas are uncultivated bacteria associated with plant diseases in several hundred species (Lee et al., 2000; Hogenhout et al., 2008; Bertaccini et al., 2014; Namba, 2019). In infected plants, phytoplasma cells are restricted to phloem tissues and can secrete effector proteins that cause developmental abnormalities of the hosts (Sugio et al., 2011b). Typical symptoms of phytoplasma infections include stunting, dwarfism, virescence (i.e., greening of flowers), phyllody (i.e., abnormal development of floral parts into leaf-like tissues), and witches' broom (i.e., proliferation of stems and leaves), which result in substantial agricultural losses.

For classification of these uncultivated bacteria, a system based on restriction fragment length polymorphism (RFLP) analysis of their 16S rRNA genes was developed in the 1990s (Lee et al., 1993, 1998; Gundersen and Lee, 1996) and at least 33 16S rRNA gene RFLP (16Sr) groups have been described (Zhao et al., 2009; Zhao and Davis, 2016). Later, a provisional genus-level taxon 'Candidatus Phytoplasma' was proposed to accommodate these bacteria (The IRPCM Phytoplasma/Spiroplasma Working Team - Phytoplasma taxonomy group, 2004) and at least 41 ' $\mathrm{Ca}$. P.' species have been described or proposed (Bertaccini and Lee, 2018). Based on analysis of $16 \mathrm{~S}$ rRNA and other conserved genes, phytoplasmas are divided into three major phylogenetic clusters (Hogenhout and Seruga Music, 2009; Chung et al., 2013; Seruga Music et al., 2019). Early genomics studies were mainly conducted for clusters I (i.e., 'Ca. P. asteris' of group 16SrI and 'Ca. P. australiense' of 16SrXII) (Oshima et al., 2004; Bai et al., 2006; Tran-Nguyen et al., 2008) and II (i.e., ' $C a$. P. mali' of 16SrX) (Kube et al., 2008). In comparison, cluster III harbors the highest level of diversity, yet has received limited attention for comparative genomics studies (Chung et al., 2013; Wang et al., 2018a).

To improve our understanding of phytoplasma genome diversity, we conducted whole genome sequencing of a ' $\mathrm{Ca}$. P. luffae' strain collected in Taiwan. The species-level taxon 'Ca. P luffae' belongs to group 16SrVIII in cluster III and is associated with witches' broom disease of loofah ( $\mathrm{Luffa}$ aegyptiaca) (Davis et al., 2017). The availability of a complete genome sequence from this taxon provides a complete view of its gene content, which can facilitate the study of its pathogenesis mechanisms and other aspects of its biology. More importantly, with the increased availability of genome sequences from diverse phytoplasmas (Table 1), we performed genus-level comparative analysis to obtain a more comprehensive picture of their genomic diversity. This improves upon previous works that are limited to comparisons of closely related taxa or have sparse sampling (Bai et al., 2006; Tran-Nguyen et al., 2008; Kube et al., 2012;
Saccardo et al., 2012; Andersen et al., 2013; Chung et al., 2013; Orlovskis et al., 2017; Wang et al., 2018a; Cho et al., 2019, 2020a; Seruga Music et al., 2019; Kirdat et al., 2021; Zhao et al., 2021). Furthermore, our focused analysis of the potential mobile units (PMUs) (Bai et al., 2006) revealed the influential roles of these mobile genetic elements in the evolution of phytoplasma genome organization and effector gene content.

\section{MATERIALS AND METHODS}

\section{Biological Materials}

The strain NCHU2019 was collected from a naturally infected loofah plant found in Dacheng Township (Changhua County, Taiwan; 23.861860 N, 120.291322 E) on July 4, 2019 (Figure 1A). After initial collection, the bacterium was transferred to labgrown loofah plants (cultivar A-Jun, Known-You Seed Co., Kaohsiung, Taiwan) through grafting and maintained in a plant growth facility in National Chung Hsing University (Taichung, Taiwan) (Figure 1B). To confirm the presence and identity of this phytoplasma strain, a partial sequence of the rRNA operon was PCR amplified using the phytoplasma-specific primer set P1/P7 for Sanger sequencing as described (Chung et al., 2013).

\section{Genome Sequencing, Assembly, and Annotation}

The procedures for genome sequencing and analysis were based on those described in our previous work on phytoplasma genomes (Chung et al., 2013; Cho et al., 2019, 2020a,b). All kits were used according to the manufacturer's protocols and all bioinformatics tools were used with the default settings unless stated otherwise.

For whole genome shotgun sequencing, leaves from one artificially infected plant exhibiting typical symptoms (i.e., small leaves and witches' broom) (Figure 1B) were collected for total genomic DNA extraction using the Wizard Genomic DNA Purification Kit (A1120; Promega, Madison, WI, United States). For Illumina sequencing, the DNA sample was processed using the KAPA Library Preparation Kit (KK8234) and the Invitrogen SizeSelect Gels (G6610-02) to obtain 550 bp fragments, followed by MiSeq $2 \times 300$ bp paired-end sequencing (v3 chemistry). For Oxford Nanopore Technologies (ONT) sequencing, the library was prepared using the ONT Ligation Kit (SQK-LSK109) without shearing or size selection, followed by MinION sequencing (R9.4.1 chemistry) and Guppy v3.3.3 basecalling.

The de novo genome assembly involved two stages. In the first stage, only the Illumina reads were used for running Velvet v1.2.10 (Zerbino and Birney, 2008) with k-mer length set to 
TABLE 1 | List of the genome assemblies analyzed. For each strain, information regarding the 16S rRNA gene (16Sr) group, genome accession number, assembly status, genome size, coding sequence (CDS) count, potential mobile unit (PMU) gene count, and combined length of PMU genes are provided. The PMU gene information is based on homologs of eight core genes (i.e., tra5, dnaB, dnaG, tmk, hflB, himA, ssb, and rpoD), including full-length genes and putative pseudogenes. A

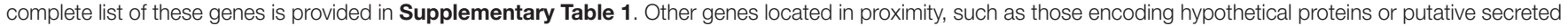
proteins, are excluded. Acholeplasma laidlawii is included as an outgroup.

\begin{tabular}{|c|c|c|c|c|c|c|c|}
\hline Strain & 16Sr group & Accession & Assembly & Genome size (bp) & CDS count & PMU gene count & PMU gene length (bp) \\
\hline 'Ca. P. asteris' AYWB & 1 & GCF_000012225.1 & Complete & 706,569 & 514 & 112 & 49,188 \\
\hline 'Ca. P. asteris' De Villa & 1 & GCF_004214875.1 & Complete & 600,116 & 521 & 21 & 11,958 \\
\hline 'Ca. P. asteris' DY2014 & 1 & GCA_005093185.1 & 8 contigs & 824,596 & 775 & 89 & 48,761 \\
\hline 'Ca. P. asteris' LD1 & I & GCF_001866375.1 & 8 contigs & 599,264 & 513 & 29 & 15,441 \\
\hline 'Ca. P. asteris' M3 & I & GCF_001712875.1 & Complete & 576,118 & 490 & 27 & 14,541 \\
\hline 'Ca. P. asteris' OY-M & 1 & GCF_000009845.1 & Complete & 853,092 & 708 & 137 & 72,399 \\
\hline 'Ca. P. tritici' WBD & 1 & GCF_000495255.1 & 6 contigs & 611,462 & 471 & 45 & 19,549 \\
\hline 'Ca. P. aurantifolia' NCHU2014 & $\|$ & GCA_001307505.2 & Complete & 635,584 & 471 & 23 & 16,378 \\
\hline 'Ca. P. pruni' CX & III & GCF_001277135.1 & 46 contigs & 598,511 & 434 & 31 & 22,474 \\
\hline 'Ca. P. ziziphi' Jwb-nky & V & GCF_003640545.1 & Complete & 750,803 & 641 & 69 & 49,824 \\
\hline 'Ca. P. luffae' NCHU2O19 & VIII & GCA_018024475.1 & Complete & 769,143 & 725 & 117 & 84,321 \\
\hline 'Ca. P. mali' AT & $x$ & GCF_000026205.1 & Complete & 601,943 & 495 & 20 & 15,084 \\
\hline 'Ca. P. oryzae' Mbita & $X I$ & GCF_001578535.1 & 28 contigs & 533,195 & 432 & 17 & 14,259 \\
\hline 'Ca. P. sacchari' SCGS & $X I$ & GCF_009268105.1 & 28 contigs & 502,197 & 402 & 15 & 13,620 \\
\hline 'Ca. P. australiense' NZSb11 & $X I I$ & GCF_000397185.1 & Complete & 959,779 & 828 & 131 & 78,642 \\
\hline 'Ca. P. australiense' PAa & XII & GCF_000069925.1 & Complete & 879,959 & 699 & 108 & 55,565 \\
\hline 'Ca. P. solani' SA-1 & XII & GCA_003698095.1 & 19 contigs & 821,322 & 709 & 66 & 45,234 \\
\hline 'Ca. P. cynodontis' LW01 & XIV & GCF_009268075.1 & 23 contigs & 483,935 & 425 & 13 & 11,856 \\
\hline ‘Ca. P. pini’ MDPP & $X X I$ & GCF_007821455.1 & 16 contigs & 474,136 & 392 & 15 & 11,685 \\
\hline Acholeplasma laidlawii PG-8A & $\mathrm{N} / \mathrm{A}$ & GCF_000018785.1 & Complete & $1,496,992$ & 1,378 & 10 & 8,811 \\
\hline
\end{tabular}
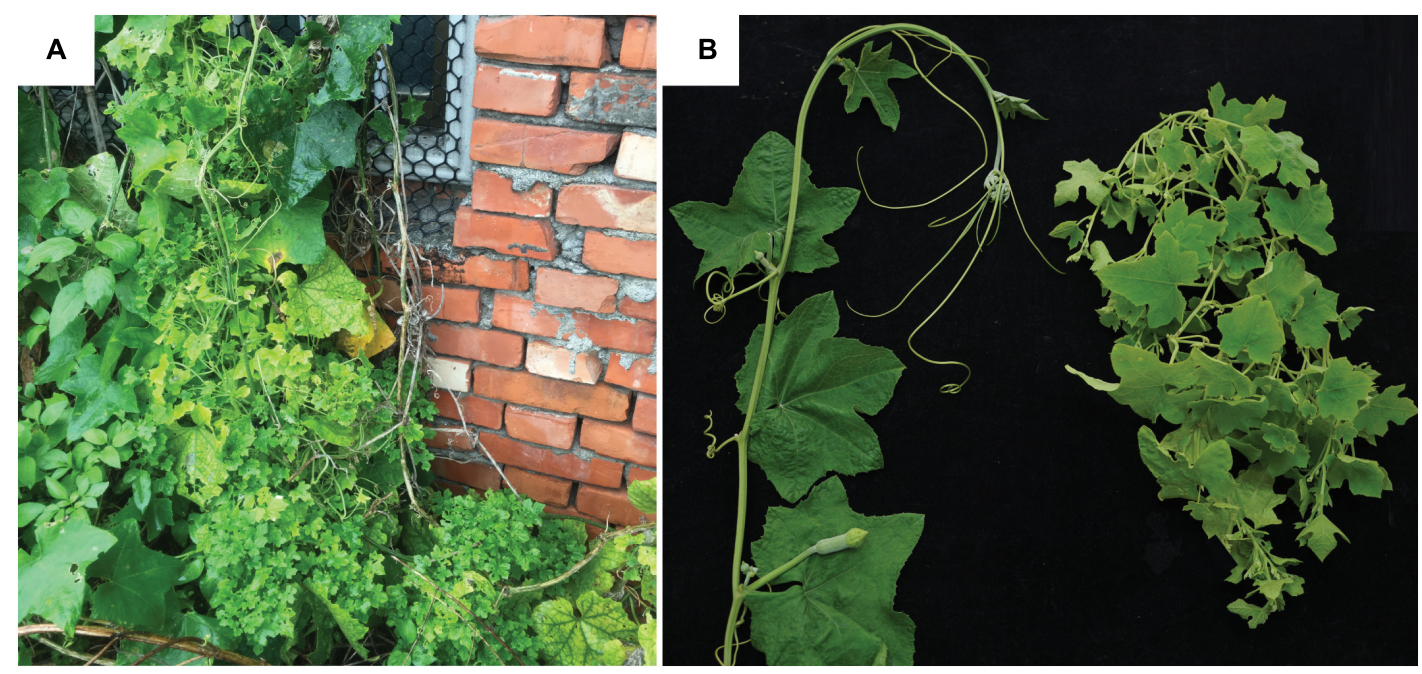

FIGURE 1 | Infection symptoms. (A) The loofah plant exhibiting phytoplasma infection symptoms that was collected in Changhua, Taiwan. (B) Loofah plants grown in the lab. Left, healthy control; right, grafted with the infected plant shown in panel (A).

151 and minimum contig length set to 2,000 bp. To identify putative phytoplasma contigs, all contigs were queried against the NCBI non-redundant protein database (Benson et al., 2018) using BLASTX v2.10.0 (Camacho et al., 2009). Those having a hit with an $e$-value of $<1 e-15$ to phytoplasma proteins were selected for manual inspection. False positive contigs derived from plant chloroplast and mitochondrial genomes were identified based on the difference in sequencing depth and verified based on BLASTN v2.10.0 (Camacho et al., 2009) searches against the NCBI standard nucleotide collection database (Benson et al., 2018).

To start the second stage of assembly, the putative phytoplasma contigs identified from the first stage were used as the reference for extracting phytoplasma reads from the Illumina data set using BWA v0.7.17 (Li and Durbin, 2009) with an alignment score cutoff of 30 and from the ONT data set using Minimap2 v2.15 (Li, 2018) with an alignment score 
cutoff of 1,000. The extracted reads were processed together for de novo hybrid assembly using Unicycler v0.4.9b. The contigs were validated as belonging to the phytoplasma genome by BLAST searches according to the process utilized in the first stage and used as the starting point for an iterative process of assembly improvement. In each iteration, all Illumina and ONT raw reads were mapped to the draft assembly as described. The mapping results were programmatically checked using the "mpileup" function of SAMTOOLS v1.9 (Li et al., 2009) and manually inspected using IGV v2.5.0 (Robinson et al., 2011) to identify possible assembly errors. Regions with raw reads mapping results exhibiting abnormalities were cut and re-arranged manually based on the continuity of ONT long reads, then validated using the mapping results in the next iteration. During the early iterations, the mapping results of ONT reads were used to provide scaffolding information and validate the overall organization of the circular chromosome, particularly the junctions between repetitive and unique regions. Additionally, the reads mapped to contig ends were visually inspected using IGV for manual selection of representative reads that can extend contigs and fill gaps. During the later iterations, the mapping results of Illumina reads were used to validate bp-scale indels and possible sequencing errors introduced by the ONT reads. The process was repeated until the complete genome assembly was obtained and all regions are supported by the raw reads mapping results. Additionally, the "depth" function of SAMTOOLS is used to calculate the sequencing coverage.

To provide a genome size estimate based on $\mathrm{k}$-mer distribution, all Illumina reads mapped to the finalized assembly with an alignment score above 200 were extracted. Based on these reads, occurrences of k-mers in the size range between 17 and 63 were calculated by using jellyfish v2.2.8 (Marçais and Kingsford, 2011). The genome size was estimated by dividing the total k-mer count with the peak depth as suggested previously (Lu et al., 2016).

The procedure of gene prediction was performed using RNAmmer v1.2 (Lagesen et al., 2007), tRNAscan-SE v1.3.1 (Lowe and Eddy, 1997), and Prodigal v2.6.3 (Hyatt et al., 2010). The annotation was based on the homologous gene clusters present in other phytoplasma genomes (Table 1) as identified by BLASTP v 2.10.0 (Camacho et al., 2009) and OrthoMCL v1.3 (Li et al., 2003), followed by manual curation based on information obtained from GenBank (Benson et al., 2018), KEGG (Kanehisa et al., 2010), and COG (Tatusov et al., 2003) databases. Additionally, putative secreted proteins were predicted using SignalP v5.0 (Armenteros et al., 2019) based on the Grampositive bacteria model. Those candidates with transmembrane domains identified by TMHMM v2.0 (Krogh et al., 2001) were removed and the remaining ones were required to have a signal peptide length in the range of 21-52 amino acids. For visualization, the Circos v0.69-6 (Krzywinski et al., 2009) was used to draw the genome map.

\section{Comparative Analysis}

For comparative analysis with other representative phytoplasma genomes (Table 1), homologous gene clusters were identified using OrthoMCL (Li et al., 2003). Multiple sequence alignments of homologous genes were prepared using MUSCLE v3.8.31 (Edgar, 2004) and visualized using JalView v2.11 (Waterhouse et al., 2009). Maximum likelihood phylogenies were inferred using PhyML v3.3 (Guindon and Gascuel, 2003) with the default LG substitution model and visualized using FigTree v1.4.4. PHYLIP v3.697 (Felsenstein, 1989) was used for bootstrap analysis. Classification of the 16S RFLP group was performed using $i$ PhyClassifier (Zhao et al., 2009).

For whole-genome comparison, FastANI v1.1 (Jain et al., 2018) was used to calculate the proportion of genomic segments mapped and the average nucleotide identity (ANI). For pairwise genome alignments, Mummer v3.23 (Kurtz et al., 2004) was used with the options "-maxmatch -mincluster 30" and the results were visualized using genoPlotR v0.8.9 (Guy et al., 2010).

The PMU analysis was based on the eight core genes (i.e., tra5, $d n a B, d n a G, t m k, h f l B, \operatorname{him} A, s s b$, and $r p o D)$ defined previously (Bai et al., 2006). To ensure uniform annotation and to include possible pseudogenes, all genome assemblies were examined by using representative PMU genes as queries to run TBLASTN searches. Hits that have a high-scoring segment pair (HSP) accounting for at least $30 \%$ of the query length and at least $50 \%$ sequence similarity within HSP were identified (Table 1 and Supplementary Table 1 ). For defining a putative PMU region, at least four intact core genes are required to be in proximity and have the same orientation. This criterion was chosen to identify the less degenerated PMU regions for the purpose of establishing a classification scheme. The limitation is that the more fragmented PMU regions would be excluded from the analysis. Exceptions were made for two putative PMU regions that do not meet this criterion, namely, AYWB_4 and AYWB_5, because ' $C a$. P. asteris' AYWB is often used as a reference for comparative analysis of PMUs. All statistical tests were performed in the R statistical environment (R Core Team, 2019); correlation coefficients were calculated using the "cor.test" function, linear regression was performed using the " $\mathrm{lm}$ " function and visualized using the "plot" and "abline" functions.

\section{RESULTS AND DISCUSSION}

\section{Genomic Characterization of NCHU2019}

The shotgun sequencing generated $\sim 4.7 \mathrm{~Gb}$ of Illumina raw reads and $\sim 3.5 \mathrm{~Gb}$ of ONT raw reads. In the first stage of de novo assembly with only the Illumina reads, a draft assembly with 49 contigs totaling 450,754 bp was obtained (longest contig: 31,507 bp; shortest contig: 2,001 bp; N50: 12,506 bp). Based on the mapping results to this first draft assembly, we extracted $0.9 \%(136,116$ out of $15,655,220)$ of all Illumina raw reads and $1.7 \%(9,416$ out of 539,354$)$ of all ONT raw reads. These reads correspond to a sequencing depth of 71 - and 110-fold, respectively. In the second stage of de novo hybrid assembly using these extracted reads from both sequencing platforms, a second draft assembly with 10 contigs totaling 668,430 bp was obtained. These 10 contigs include two long ones that are 552,199 and 103,217 bp in size, as well as eight short ones that are shorter than 3,000 bp. Examination of the mapping results to this second draft 
assembly found 186,979 Illumina reads with 73 -fold coverage and 13,242 ONT reads with 95 -fold coverage.

During the iterative assembly improvement process, a pair of duplicated chromosomal segments that are each $\sim 75 \mathrm{~kb}$ in size and multiple smaller repeats were identified. These regions were manually corrected to generate the finalized assembly. All junctions involving repetitive regions were verified based on visual inspection of the ONT long read mapping results. For the finalized assembly, we obtained a circular contig that corresponds to the phytoplasma chromosome; no plasmid was found. This circular contig is 769,143 bp in size with $23.3 \%$ $\mathrm{G}+\mathrm{C}$ content (Figure 2). For the final round of raw reads mapping examination, 149,857 Illumina reads and 13,649 ONT reads were mapped to this circular contig, corresponding to 51- and 80-fold sequencing depth, respectively. The decrease of sequencing depth compared to the draft assemblies is likely explained by the repetitive regions being resolved. As a support for this inference, the sequencing depth exhibits a nearly uniform distribution across the entire finalized circular contig, with repetitive regions having similar depth as non-repetitive regions (Supplementary Figure 1).

For genome size estimation based on k-mer analysis, we utilized $k=21$ as a representative data set for visualization (Supplementary Figure 2A). We found that while the peak depth is 51 , the frequency distribution is nearly flat in the range between $\sim 40$ and 55 . Based on this distribution, the genome size is estimated to be in the range between 660 and $842 \mathrm{~kb}$ (Supplementary Figure 2B). The assembled genome size of $769 \mathrm{~kb}$ is near the middle point (i.e., $751 \mathrm{~kb}$ ) of these estimates.

The annotation of this phytoplasma genome contains two complete sets of 16S-23S-5S rRNA gene clusters, 31 tRNA genes, 725 coding sequences (CDSs), and 13 pseudogenes. Both copies of the 16S rRNA gene are $100 \%$ identical to the reference sequence of 'Ca. P. luffae' LfWB (GenBank accession AF248956). Among the CDSs, 317 (44\%) lacked any COG functional category assignments. Among the CDSs that were assigned to specific functional categories, those assigned to information storage and processing (e.g., replication, transcription, and translation) represent the largest group that account for $32 \%$ of all CDSs. In comparison, those assigned to cell process and metabolism account for 9 and $13 \%$, respectively. These observations are consistent with findings from characterization of other phytoplasmas and more distantly related Mollicutes (e.g., Spiroplasma, Entomoplasma, and Mycoplasma) (Chen et al., 2012; Kube et al., 2012; Lo et al., 2018). The observation that a large fraction of genes lack specific functional annotation may be attributed to the elevated evolutionary rates of Mollicutes and their distant phylogenetic relationships from model organisms (Wu and Eisen, 2008; Wu et al., 2009; McCutcheon and Moran, 2012). The observation that genes related to information storage and processing genes are relatively abundant compared to those involved in metabolism is common among symbiotic bacteria with small genomes (McCutcheon and Moran, 2012; Lo et al., 2016).

Other than the low $\mathrm{G}+\mathrm{C}$ content and reduced gene content, phytoplasma genomes are generally known to be repetitive, partly due to the presence of PMUs (Sugio and Hogenhout, 2012).
Interestingly, the genome of this strain is far more repetitive than other phytoplasmas that have been characterized. On average, PMU core genes account for $\sim 4.7 \%$ (Std. Dev. $=2.7 \%$ ) of the genome size among those 19 representative phytoplasma strains analyzed (Table $\mathbf{1}$ and Supplementary Table 1). For strain NCHU2019, there are 117 PMU core genes organized into at least 13 distinct PMU regions (Figure 2 and Supplementary Table 2) and account for $11 \%$ of the chromosome length. Additionally, a pair of chromosomal segments, each $\sim 75 \mathrm{~kb}$ in size, were found to be duplicated (positions: $315,975-391,140$ and 391,446466,612; sequence identity: $75,160 / 75,167=99.99 \%)$. The same set of 79 CDSs arranged in the same order were found in each of the repeats. Out of these CDSs, 27 correspond to hypothetical proteins. Two notable genes are $\operatorname{ruv} A / B$, which are involved DNA recombination and repair. The duplication of these genes made NCHU2019 the only phytoplasma strain with two copies of $\operatorname{ruv} A / B$ reported to date. Together, these two duplicated segments and the 13 PMU regions account for $25 \%$ of the chromosome length. Explanation for the high genome repetitiveness of strain NCHU2019 compared to other phytoplasmas is unclear.

\section{Comparisons With Other Phytoplasmas}

Based on the established classification scheme and a previous study of 16S rRNA gene phylogeny (Davis et al., 2017), 'Ca. P. luffae' belongs to group 16SrVIII and is most closely related to 'Ca. P. malaysianum' (group 16SrXXXII; GenBank accession EU371934) (Nejat et al., 2013). These two specieslevel taxa have $95.9 \%$ sequence identity (i.e., 1,463/1,526 aligned nucleotides) in their $16 \mathrm{~S}$ rRNA genes. However, no genome sequence is available for ' $\mathrm{Ca}$. P. malaysianum' for comparative analysis.

Other than ' $\mathrm{C} a$. P. malaysianum, the next closest relatives of 'Ca. P. luffae' include phytoplasmas belonging to groups $16 \mathrm{SrV}$ ('Ca. P. ziziphi'), 16SrVI ('Ca. P. sudamericanum' and 'Ca. P. trifolii'), and $16 \mathrm{SrVII}$ ('Ca. P. fraxini'). Among these, one complete genome sequence (GenBank accession CP025121) is available for ' $\mathrm{Ca}$. P. ziziphi' strain Jwb-nky (Wang et al., 2018a), which represents the most closely related lineage for comparative analysis (Figure 3A). Comparison based on the $16 \mathrm{~S}$ rRNA gene sequences indicated that ' $\mathrm{Ca}$. P. luffae' NCHU2019 and 'Ca. P. ziziphi' Jwb-nky have $94.9 \%$ sequence identity (i.e., $1,447 / 1,524$ aligned nucleotides). For whole-genome comparison, only $49 \%$ of the chromosomal segments can be mapped between these two strains and these segments have $<80 \%$ ANI. Pairwise genome alignment indicated that the most of the conserved regions correspond to PMUs (Figure 4), which further supports that the sequence divergence between these two genomes is too high for nucleotide-level comparisons. The lack of chromosome-level synteny conservation was commonly reported in previous comparisons between complete genome sequences of phytoplasmas (Bai et al., 2006; Tran-Nguyen et al., 2008; Andersen et al., 2013; Orlovskis et al., 2017; Wang et al., 2018a), even for ' $\mathrm{Ca}$. P. asteris' strains sharing $>99.9 \% 16 \mathrm{~S}$ rRNA gene sequence identity and $>98.1 \%$ genome-wide ANI (Cho et al., 2020a). These observations may be attributed to the strong nucleotide composition biases, the high mutation accumulation rates, and the influence of PMUs (Cho et al., 2020a). 


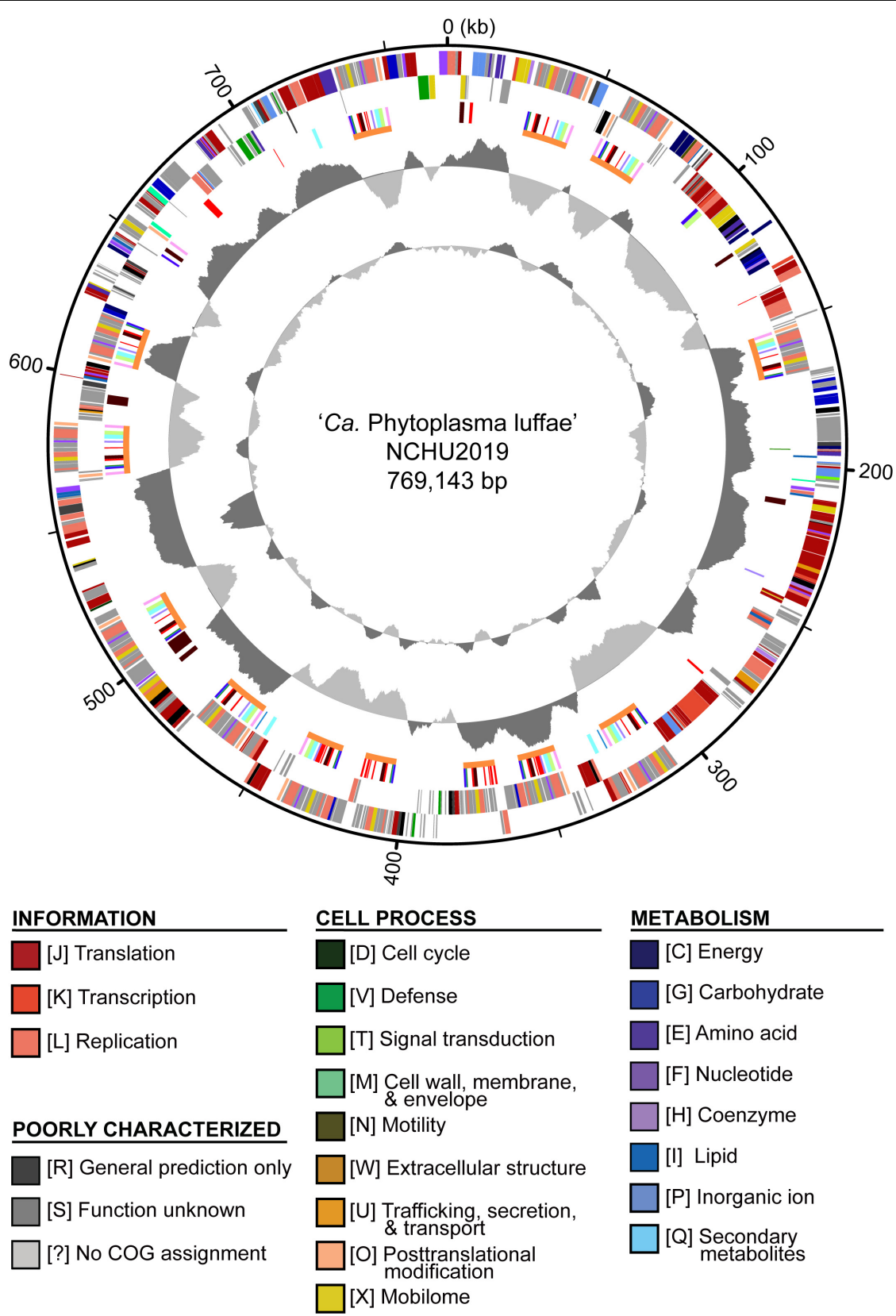

FIGURE 2 | Genome map of 'Candidatus Phytoplasma luffae' NCHU2019. Rings from outside in: (1) Scale marks (kb). (2 and 3) Coding sequences on the forward and reverse strand, respectively. Color-coded by functional categories. (4) Genes associated with potential mobile units (PMUs), color-coded by annotation (see Figure 5). Gene clusters that represent individual PMUs are labeled by orange lines. Genes encoding putative secreted proteins are in red. (5) GC skew (positive: dark gray; negative: light gray). (6) GC content (above average: dark gray; below average; light gray). One high GC peak located in the $531-544$ kb region corresponds to two adjacent rRNA operons.

Considering the high level of nucleotide sequence divergence between ' $C a$. P. luffae' NCHU2019 and ' $C a$. P. ziziphi' Jwbnky, we also examined gene content based on protein sequence comparisons. In the pairwise comparison, 344 homologous gene clusters are shared between these two strains, while 175 and 241 are specific to NCHU2019 and Jwb-nky, respectively. These counts of shared and strain-specific genes are comparable to those found in previous studies of phytoplasma genome comparisons at between-species level (Chung et al., 2013; Tan et al., 2021). Although relatively large numbers of strain-specific genes were identified, $>80 \%$ of these genes lack functional annotation, making the inference of their roles difficult. As such, the gene content regarding metabolic capacity and transporters of ' $\mathrm{Ca}$. P. luffae' NCHU2019 is expected to be highly similar to that of 'Ca. P. ziziphi' Jwb-nky, which was described in detail previously (Wang et al., 2018a). 


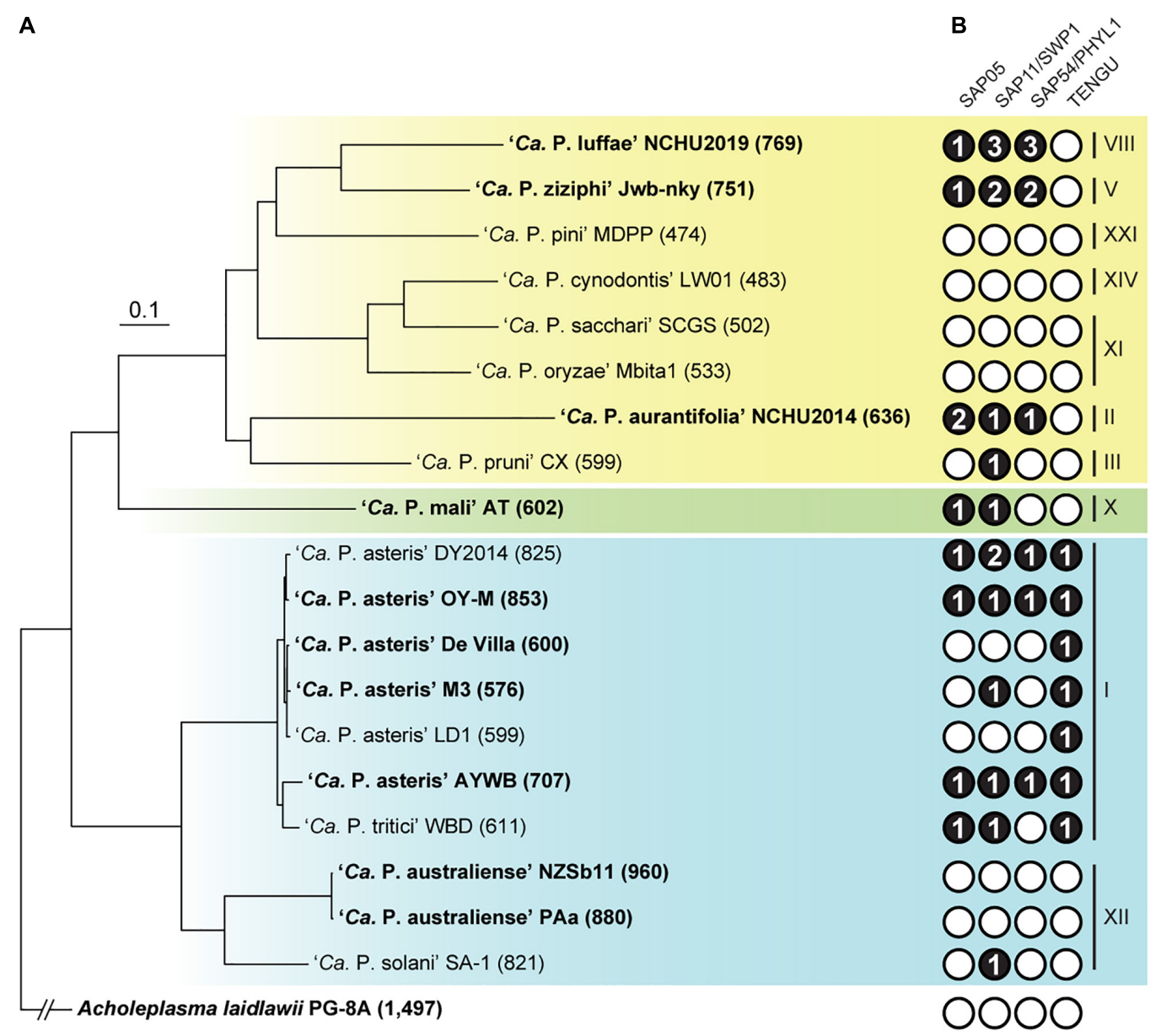

FIGURE 3 | Evolutionary relationships among representative phytoplasmas with genome sequences available. The 16S rRNA gene (16Sr) group assignments are labeled on the right. The three major phylogenetic clusters are indicated by colored backgrounds (I: blue; II; green; III: yellow). Acholeplasma laidlawii is included as the outgroup. (A) Maximum likelihood phylogeny inferred based on a concatenated alignment of 80 conserved single-copy genes (27,742 aligned amino acid sites). All internal nodes received $>80 \%$ bootstrap support based on 1,000 re-sampling. Strains with complete genome assemblies available are highlighted in bold. The number in parentheses following the strain name indicates the genome size (in kb). (B) Distribution of known effector genes. Gene presence and absence are indicated by filled (with copy numbers) and empty circles, respectively.

For comparisons with those more divergent phytoplasmas with genomic information available (Table 1), only 134 homologous gene clusters are conserved among the 19 representatives analyzed. This estimate of phytoplasma core genome is much lower than the $\sim 200$ genes consistently reported in previous studies (Chen et al., 2012; Chung et al., 2013; Tan et al., 2021), an observation that is likely explained by the inclusion of several draft assemblies in this study. Detailed description regarding the functions of these $\sim 200$ core genes were reported previously (Chen et al., 2012; Kube et al., 2012; Chung et al., 2013).

\section{Detailed Characterization of Potential Mobile Units}

To better understand the roles of PMUs in phytoplasma genome evolution, we conducted detailed characterization of these mobile genetic elements. Among the 13 PMUs identified in ' $\mathrm{Ca}$. P. luffae'
NCHU2019 (Figure 5A), 11 are considered as complete ones and range from 14 to $18 \mathrm{~kb}$ in size. The remaining two (i.e., \#6 and \#7) are both $12 \mathrm{~kb}$ in size and appear to be truncated based on the lack of multiple PMU core genes (i.e., tmk, dnaB, dnaG, and tra5). These 13 PMUs are variable in the gene content in between rad50 and tmk, while the sequences of shared core genes are highly conserved. For example, the tmk and $d n a B$ homologs among these PMUs have identical sequences (Figure 6). It is unclear if the sequence conservation of these core genes is explained by lack of mutation accumulation, purifying selection, or assembly artifacts. Regarding chromosomal locations, these PMUs are interspersed across the entire chromosome and there is no obvious pattern of clustering (Figures 2, 4). Two sets of PMUs (i.e., \#4-6 and \#7-9) are associated with the two $75-\mathrm{kb}$ repeat regions. Notably, one set of junctions for these large repeats (chromosomal positions 315,975 and 466,612) are located inside PMU \#4 (positions 308,032.323,983) and PMU \#9 (positions 458,604.475,923). This finding suggests 


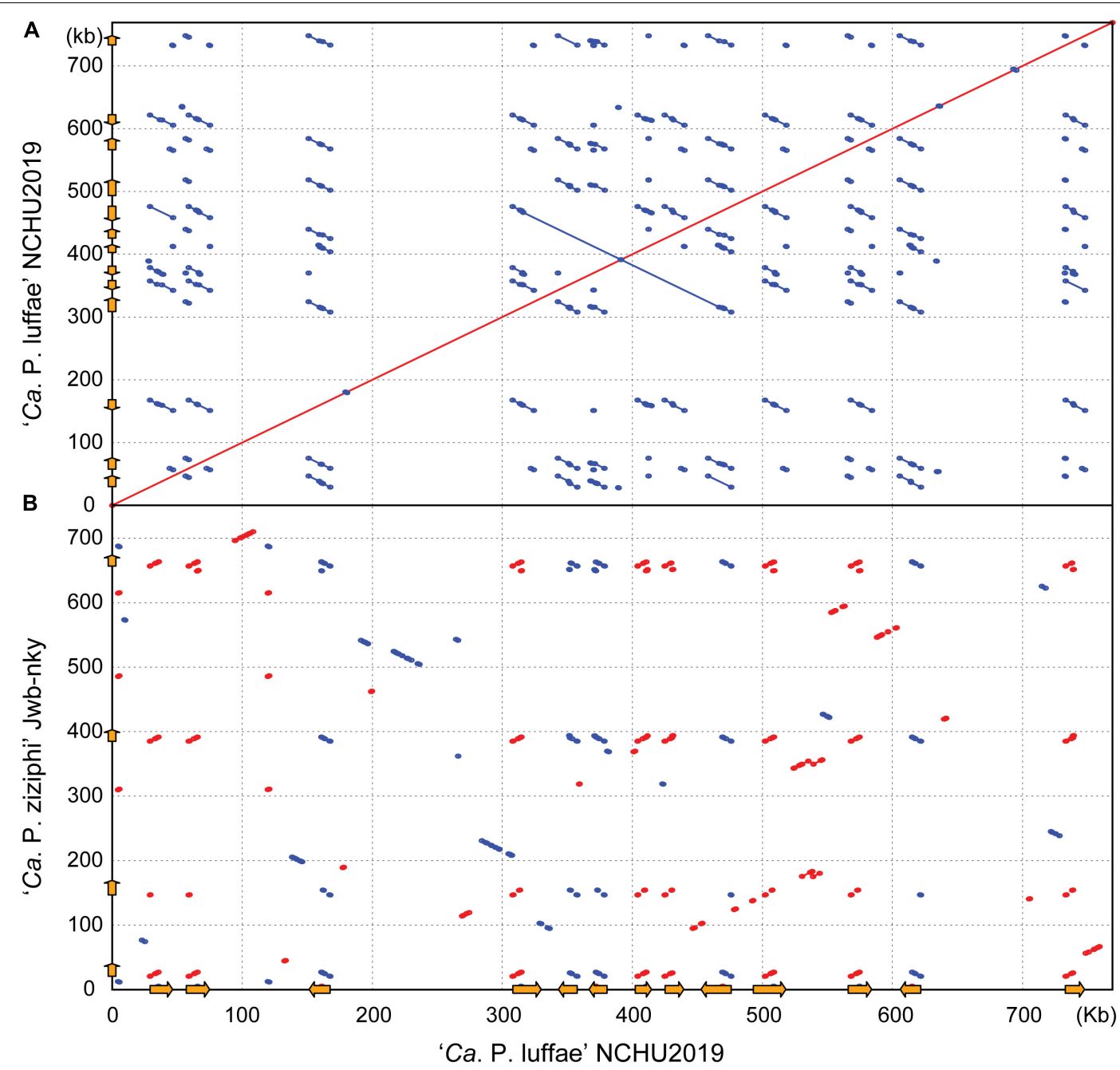

FIGURE 4 | Pairwise genome alignments. The genome of 'Ca. P. luffae' NCHU2019 was used as the reference for comparison with (A) itself and (B) 'Ca. P. ziziphi' Jwb-nky. Matches on the same strand and the opposite strand are indicated in red and blue, respectively. Potential mobile units (PMUs) are illustrated as orange arrows on both axes.

that homologous recombination facilitated by PMUs may have promoted the segmental duplication of this chromosome, which increased the PMU copy number.

Based on initial characterization of the PMUs in ' $\mathrm{Ca}$. P. asteris' AYWB genome, eight core genes were identified (Bai et al., 2006). As more genome sequences become available from diverse phytoplasmas, we were able to include representatives of 13 ' $\mathrm{Ca}$. P.' species from all three phylogenetic clusters (Table 1) for comparison. Compared to other phytoplasmas, ' $\mathrm{Ca}$. P. luffae' NCHU2019 likely has the highest number of intact PMUs and is distinctive in that all of its PMUs are similar (Figure 5A and Supplementary Table 2). Among the 11 complete PMUs in this genome, the eight PMU core genes and rad50 are all organized in the same order. The minor variation in gene organization is mainly in between rad50 and tmk, where genes encode different putative secreted proteins and hypothetical proteins are found. For comparison, in the closely related ' $\mathrm{Ca}$. P. ziziphi' Jwb-nky of cluster III (i.e., the yellow clade in Figure $\mathbf{3 A}$ ), the four
PMUs exhibit much higher levels of diversity in gene organization between $h f l B$ and $t m k$ (Figure 5A). For the distantly related ' $C a$. P. asteris' AYWB and 'Ca. P. australiense' PAa of cluster I (i.e., the blue clade in Figure 3A), high levels of intra-genomic PMU diversity are observed (Figure 5B and Supplementary Table 2).

Based on the presence/absence pattern and order of eight PMU core genes defined previously (Bai et al., 2006), we found that the PMUs in these phytoplasmas can be classified into three major types (Figure 5B). The strains omitted in the visualization all have close relatives belonging to the same $16 \mathrm{Sr}$ group and share similar PMUs when there are identifiable ones (Supplementary Table 2). Among the three major types, type A PMUs, in which $t m k$ is upstream of $d n a B$, are the first reported ones (Bai et al., 2006) and also the most common ones that are found in phylogenetic clusters I (e.g., 'Ca. P. asteris,' 'Ca. P. australiense', and 'Ca. P. solani') and III (e.g., 'Ca. P. luffae,' 'Ca. P. ziziphi', and ' $\mathrm{Ca}$. P. aurantifolia'). Type B PMUs are shorter, have tmk downstream of $d n a B$, and are found only in ' $\mathrm{Ca}$. P. australiense' 


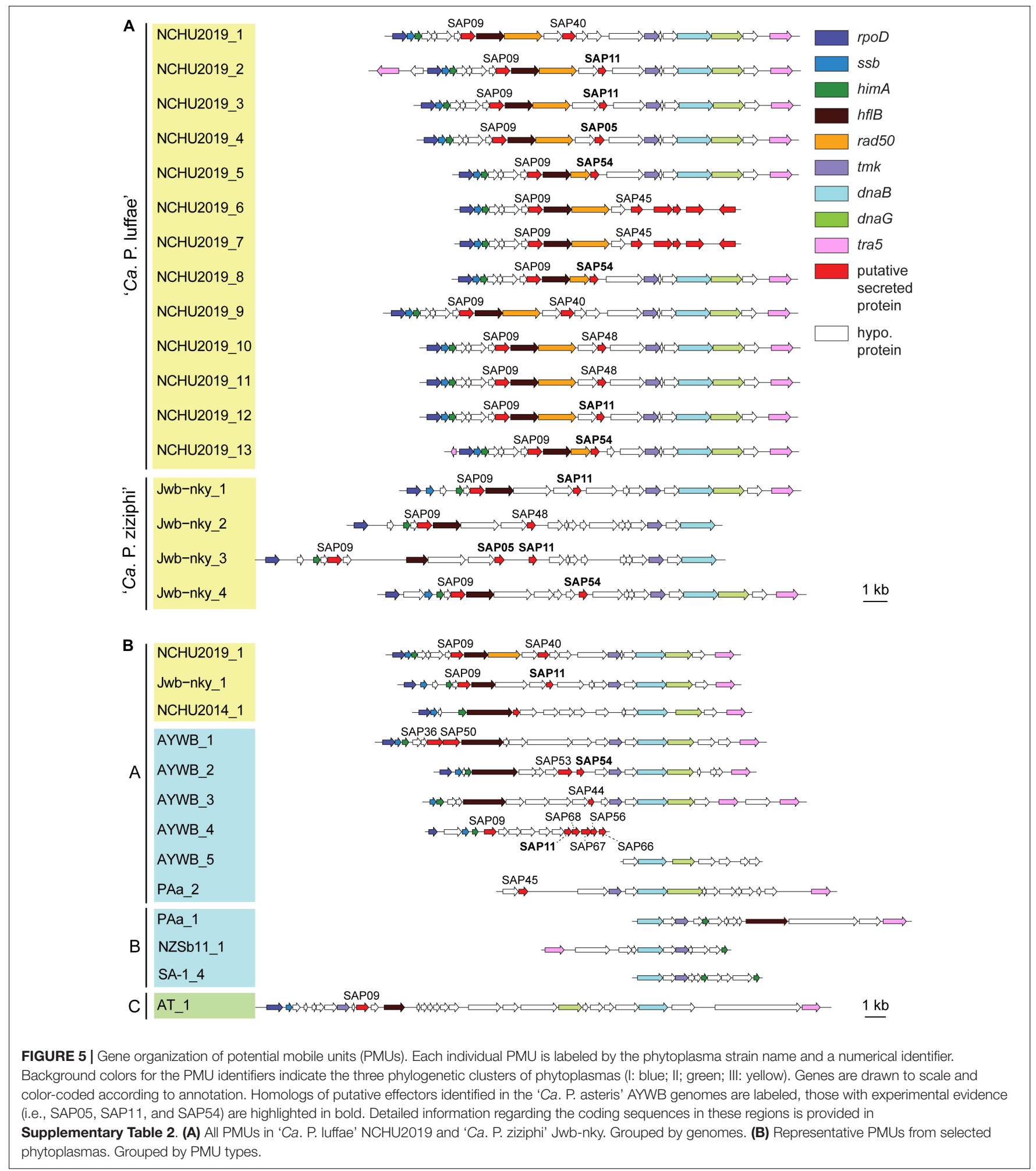

(Tran-Nguyen et al., 2008; Andersen et al., 2013) and 'Ca. P. solani' (Seruga Music et al., 2019) that belong to cluster I. Type $\mathrm{C}$ is the rarest type, with only one representative found in ' $\mathrm{Ca}$. P. mali' that belongs to cluster II (Kube et al., 2008; Chung et al., 2013), and has $h f l B$ and $d n a G$ located in between tmk and
dnaB. In addition to gene organization, molecular phylogenies of $d n a B$ and tmk also revealed sequence divergence among homologs from different PMU types (Figure 6). These patterns provide further support for our PMU classification scheme. It is interesting to note that regardless of PMU copy numbers, most 
of the phytoplasmas with genome sequences available harbor only one single type of PMUs. Two exceptions are ' $\mathrm{Ca}$. P. australiense' PAa and 'Ca. P. solani' SA-1, both harboring PMUs belong to types A and B. Future improvements in sampling more diverse phytoplasma genomes, particularly cluster II lineages, are necessary to provide a more comprehensive understanding of PMU diversity. Another point worth mentioning is that several of the genome sequences examined lack any identifiable PMU region according to our criterion, while still harbor multiple PMU genes (e.g., 'Ca. P. asteris' strains De Villa and M3). It is likely that the PMU regions in these genomes were degenerated through mutation accumulation.

\section{Potential Mobile Unit and Phytoplasma Genome Size Variation}

One notable observation regarding phytoplasma genomes is the extensive size variation at both between- and within-species levels (Table 1). Phylogenetic relatedness, as quantified by core genome sequence divergence, does not appear to provide a reliable predictor for genome sizes (Figure 3A). Previous within-species comparisons suggested that PMU abundance is an important factor for genome size variation (Bai et al., 2006; Andersen et al., 2013). To further test if this pattern holds true for genuslevel analysis, we performed regression analysis to examine the correlation between the combined length of PMU core genes and genome size. The main reason of utilizing PMU core genes, rather than putative PMU regions, is because that several genomes (e.g., 'Ca. P. asteris' OY-M) harbor putative PMU regions with highly variable degrees of fragmentation and the exact boundaries of those regions are difficult to be defined unambiguously. Furthermore, even for ' $\mathrm{Ca}$. P. luffae' NCHU2019, in which the 13 PMU regions are well defined, there are quite a few orphan PMU genes located outside of those 13 regions and scatter across the entire chromosome (Figure 2). As such, analysis based on the PMU core genes provides a more objective and accurate quantification for the relative abundance of PMUs. Strikingly, when all 19 representative phytoplasma genome assemblies were examined together, the combined length of PMU core genes explains $79 \%$ of the variance in genome sizes $(r=0.89, p=3.7 \mathrm{e}-07)$ (Figure 7$)$. Due to the concern that draft assemblies cannot provide accurate information regarding these two metrics, we also performed regression analysis with only the 10 complete assemblies and obtained similar results $(r=0.87$, $p=9.7 \mathrm{e}-04)$.

The evolution of bacterial genome sizes is a topic that received much research attention and previous studies have identified multiple relevant factors, such as composition of gene content, prevalence of mobile genetic elements, effective population sizes, level of mutational biases toward deletion, and ecological niches (Mira et al., 2001; Konstantinidis and Tiedje, 2004; Ochman and Davalos, 2006; Kuo and Ochman, 2009; Kuo et al., 2009; Novichkov et al., 2009; McCutcheon and Moran, 2012; Lo et al., 2016; Sabater-Muñoz et al., 2017; Weinert and Welch, 2017). Due to the complexity of bacterial genome size evolution and the multitude of factors involved in the process, the finding that one single factor (i.e., PMU abundance) having such a strong correlation with genome size among phytoplasmas is surprising. Notably, as genome reduction appears to be a common and recurring theme of symbiont evolution, the roles of PMU in genome expansion of some phytoplasmas require further investigation. Because PMUs are known to be associated with effector genes and likely can transfer horizontally between closely- (Cho et al., 2019; Seruga Music et al., 2019) or distantlyrelated phytoplasmas (Chung et al., 2013; Ku et al., 2013), the involvement of PMUs in phytoplasma effector gene content evolution is particularly important. However, it is worth noting that even though horizontal transfers of PMUs may provide novel combinations of effector gene content, PMU evolution may have been dominated by vertical inheritance or at least transfers between closely related lineages based on the observation that in most cases related lineages have similar PMUs.

\section{Effector Genes}

An important feature of phytoplasmas is their ability to modulate host plant development through small secreted proteins known as effectors (Sugio et al., 2011b; Sugio and Hogenhout, 2012). Four notable effectors have been experimentally characterized, including SAP05 (Gamboa et al., 2019; Huang and Hogenhout, 2019; Huang et al., 2021), SAP11/SWP1 (Bai et al., 2009; Sugio et al., 2011a; Lu et al., 2014; Chang et al., 2018; Wang et al., 2018c,b), SAP54/PHYL1 (MacLean et al., 2011; Maejima et al., 2014; Orlovskis and Hogenhout, 2016), and TENGU (Hoshi et al., 2009; Sugawara et al., 2013; Minato et al., 2014). The expanded genome sequence availability allowed us to investigate the phylogenetic distribution of homologous effector genes among diverse phytoplasmas. The highly variable distribution patterns suggest that the effector gene content may have rapid turnover during the diversification of phytoplasmas (Figure 3). For example, at within-species level comparison among ' $\mathrm{Ca}$. P. asteris' strains, the patterns of presence/absence and gene copy number are variable for three of these effectors (Figure 3B) although the level of core genome sequence divergence is very low as indicated by the short branch lengths (Figure 3A). This finding is consistent with our previous in-depth characterization of 16SrI phytoplasmas (Cho et al., 2020a). At genus-level, TENGU appears to be restricted to and conserved among 16SrI lineages in cluster I (i.e., ' $\mathrm{Ca}$. P. asteris' and ' $\mathrm{Ca}$. P. tritici'), while the other three effectors are variable. Based on these patterns, it is likely that TENGU originated in the most recent common ancestor (MRCA) of 16SrI phytoplasmas. However, for the other three effectors, it is unclear if the MRCA of all extant phytoplasmas harbor these genes or not. If yes, then multiple independent gene losses are required to explain the distribution of these genes among extant phytoplasmas. Alternatively, multiple independent origins, likely mediated by PMU-mediated horizontal gene transfer (Chung et al., 2013; Cho et al., 2019; Seruga Music et al., 2019), are required to explain the gene distribution pattern. Another interesting observation is that several phytoplasmas do not possess any of these four effector genes. It is likely that these phytoplasmas harbor novel effector genes that are yet to be characterized and further investigation is required to obtain a more complete picture of phytoplasma effector diversity. 


\section{A tmk}

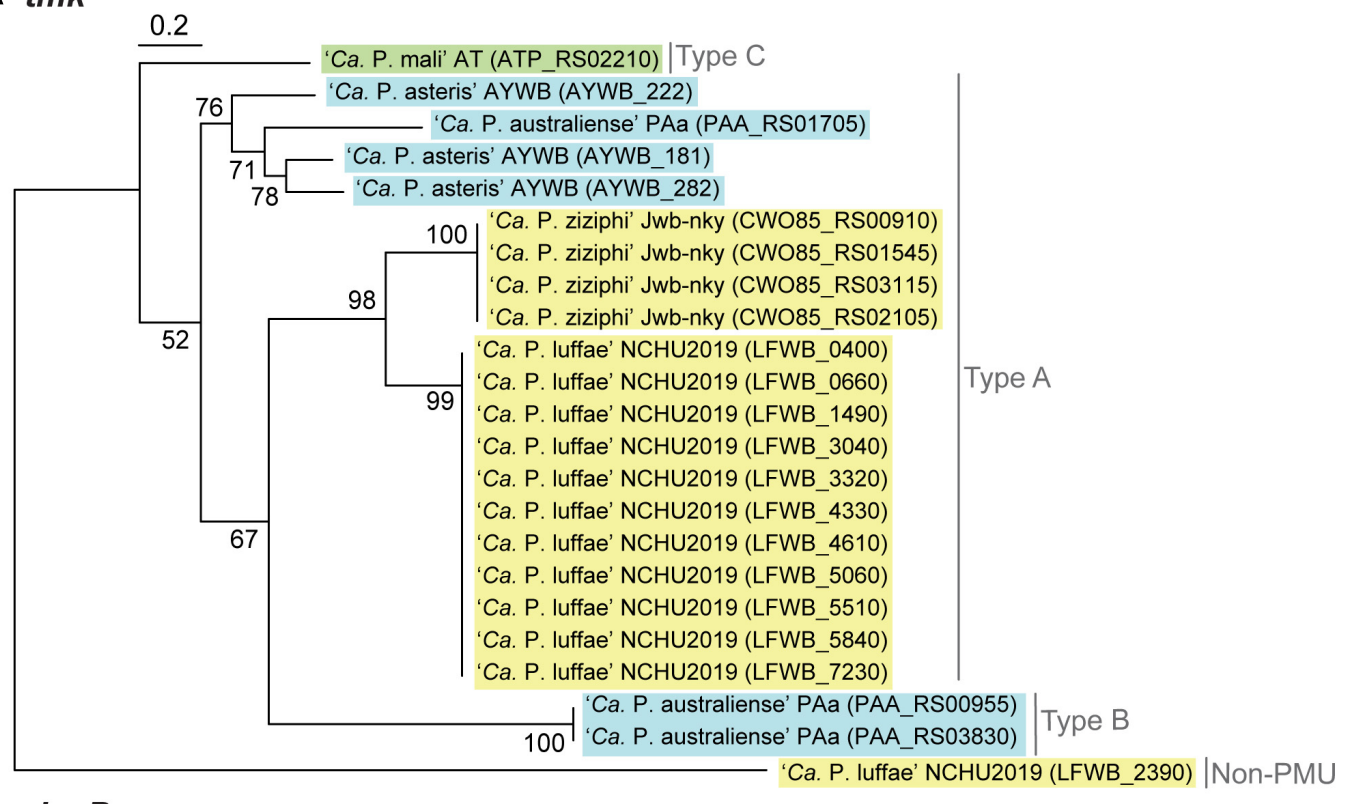

B dnaB

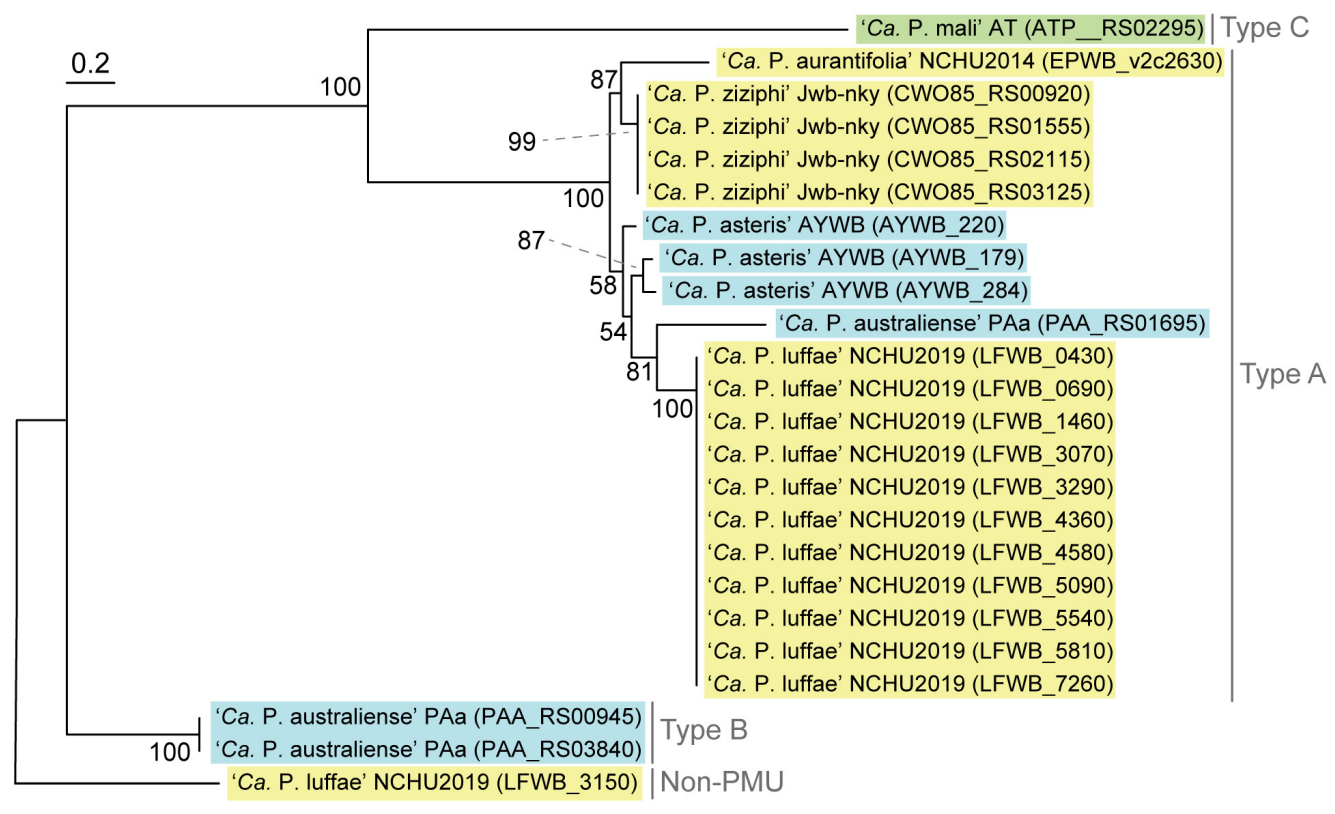

FIGURE 6 | Maximum likelihood phylogenies of PMU core genes (A) tmk (228 aligned amino acid sites). (B) dnaB (531 aligned amino acid sites). In both panels, a non-PMU homolog is included as the outgroup. Numbers next to internal branches indicate the bootstrap support levels based on 1,000 re-sampling. Background colors for the gene identifiers indicate the three phylogenetic clusters of phytoplasmas (I: blue; II; green; III: yellow).

For closer inspections of these four effectors, we performed multiple sequence alignments to examine the protein sequence divergence among homologs (Supplementary Figure 3). Consistent with the expectation derived from their phylogenetic distribution patterns, the three effector genes found among diverse phytoplasmas have higher levels of sequence divergence compared to phylogenetically restricted TENGU homologs.

For copy number variation, 'Ca. P. luffae' NCHU2019 stands out as having the highest copy numbers for SAP11 and
SAP54 (Figure 3B). These homologous genes are all located within PMUs (Figure 5) and have nearly identical sequences (Supplementary Figure 3), which suggest that recent intragenomic PMU duplications are responsible for expansions in effector gene copy numbers. Similar patterns were observed for the SAP11 and SAP54 homologs in 'Ca. P. ziziphi' Jwbnky. Experimental characterization of the two ' $\mathrm{Ca}$. P. ziziphi' SAP11 homologs demonstrated that both can stimulate lateral bud outgrowth for witches' broom symptoms when expressed 


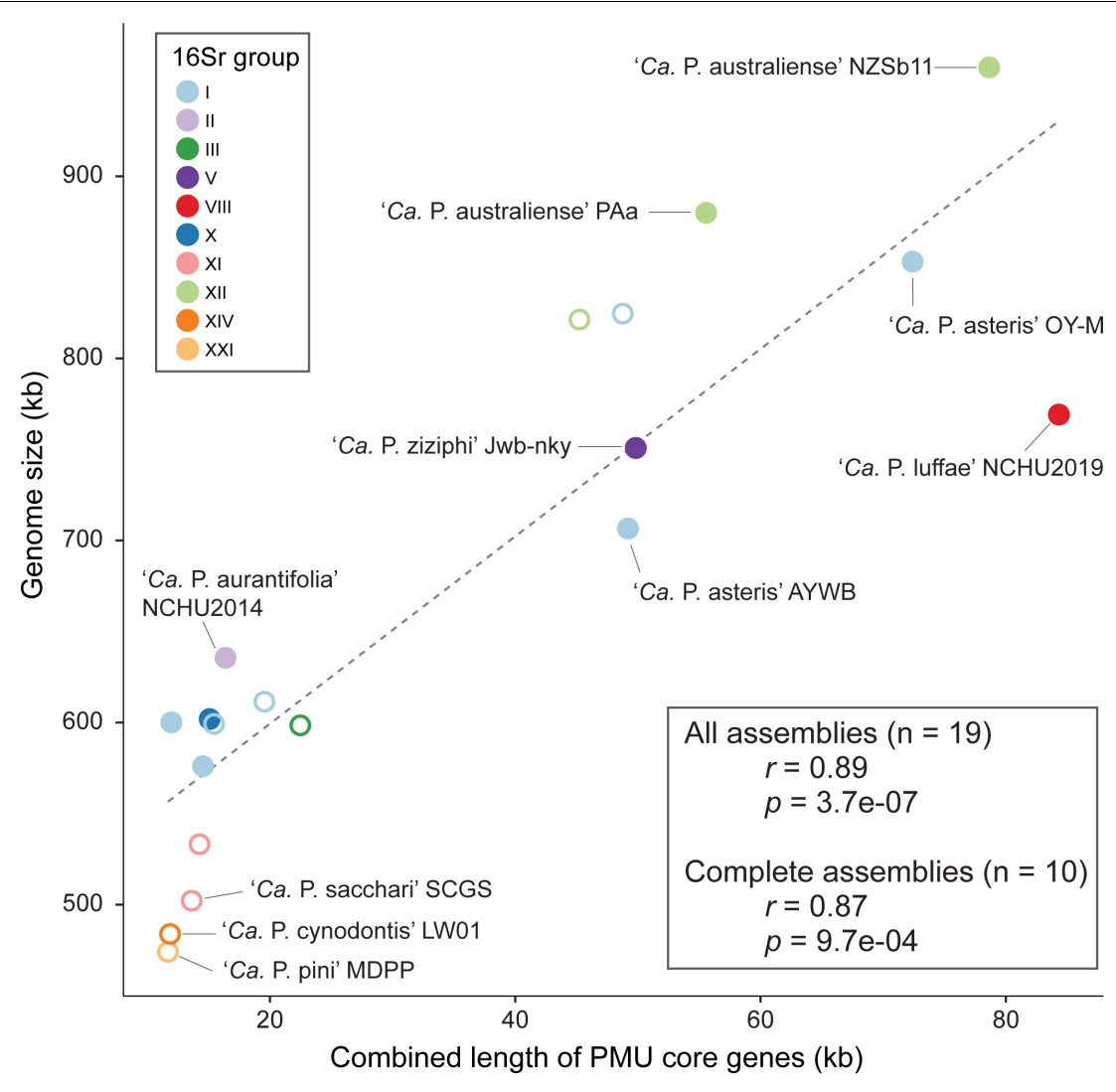

FIGURE 7 | Correlation between the combined length of PMU core genes and genome size. Strains with complete and draft genome assemblies are indicated by filled and open circles, respectively. The linear regression line was based on all available assemblies.

in Nicotiana benthamiana (Zhou et al., 2021). Intriguingly, in phytoplasma-infected jujube plants, these two SAP11 homologs have different expression patterns among tissue types. These findings suggest that such gene duplication events may lead to subfunctionalization or even neofunctionalization, thus promoting the genetic diversity of effectors.

In addition to these four effectors that have been wellcharacterized, there are likely additional effectors among diverse phytoplasmas. For example, two putative effectors were reported recently for 'Ca. P. mali' (Mittelberger et al., 2019; Strohmayer et al., 2019). The functions and evolution of these and other novel effectors warrant further studies.

\section{CONCLUSION}

In this work, we determined the complete genome sequence of an uncultivated ' $C a$. P. luffae' strain associated with the witches' broom disease of loofah. This assembly provides the first representative genome sequence for the 16SrVIII group of phytoplasmas and improves the taxon sampling of these diverse plant-pathogenic bacteria. For comparative genomics analysis conducted at genus level, we provided a global view of the PMUs (i.e., phytoplasma-specific mobile genetic elements) and identified three major PMU types that differ in gene organization and phylogenetic distribution. Importantly, statistical analysis revealed that PMU abundance explains nearly $80 \%$ of the variance in phytoplasma genome sizes, providing a quantitative estimate on the importance of these elements. Finally, our investigation of effector genes highlighted the genetic diversity associated with phytoplasma virulence and supported the roles of PMUs in shaping such diversity.

\section{DATA AVAILABILITY STATEMENT}

The datasets presented in this study can be found in online repositories. The names of the repository/repositories and accession number(s) can be found below: https://www.ncbi. nlm.nih.gov/, SRR11921288; https://www.ncbi.nlm.nih.gov/, SRR11921289; and https://www.ncbi.nlm.nih.gov/ genbank/, CP054393.

\section{AUTHOR CONTRIBUTIONS}

J-YY and C-HK conceptualized the study, acquired funding, and supervised the project. J-YY, Y-CC, and C-MT provided the biological materials. C-MT and S-TC coordinated the sequencing and conducted the initial genome assembly. S-TC completed the 
assembly. Y-CL and C-TH validated the assembly. S-TC, C-TH, and Y-CL conducted the comparative analysis and prepared the figures. C-TH and C-HK wrote the draft. All authors approved the submitted version.

\section{FUNDING}

Work in the J-YY lab was supported by grants-in-aid from the Ministry of Science and Technology (110-2628-B-005-002) and the Advanced Plant Biotechnology Center from the Featured Areas Research Center Program within the framework of the Higher Education Sprout Project by the Ministry of Education in Taiwan. Work in the C-HK lab was supported by Academia Sinica and the Ministry of Science and Technology (106-2311-B-001028-MY3) of Taiwan. The funders had no role in study design, data collection and interpretation, or the decision to submit the work for publication.

\section{ACKNOWLEDGMENTS}

We thank Ai-Ping Chen and Shu-Jen Chou for technical assistance. The Illumina sequencing library preparation service was provided by the Genomic Technology Core Facility (Institute of Plant and Microbial Biology, Academia Sinica, Taipei, Taiwan). The Illumina paired-end sequencing service was provided by the Genomics Core Facility (Institute of Molecular Biology, Academia Sinica, Taipei, Taiwan). We thank the reviewers for their helpful comments that improved this work.

\section{REFERENCES}

Andersen, M. T., Liefting, L. W., Havukkala, I., and Beever, R. E. (2013). Comparison of the complete genome sequence of two closely related isolates of 'Candidatus Phytoplasma australiense' reveals genome plasticity. BMC Genomics 14:529. doi: 10.1186/1471-2164-14-529

Armenteros, J. J. A., Tsirigos, K. D., Sønderby, C. K., Petersen, T. N., Winther, O., Brunak, S., et al. (2019). SignalP 5.0 improves signal peptide predictions using deep neural networks. Nat. Biotechnol. 37, 420-423. doi: 10.1038/s41587-0190036-z

Bai, X., Correa, V. R., Toruño, T. Y., Ammar, E.-D., Kamoun, S., and Hogenhout, S. A. (2009). AY-WB phytoplasma secretes a protein that targets plant cell nuclei. Mol. Plant. Microbe Interact. 22, 18-30. doi: 10.1094/MPMI-22-10018

Bai, X., Zhang, J., Ewing, A., Miller, S. A., Jancso Radek, A., Shevchenko, D. V., et al. (2006). Living with genome instability: the adaptation of phytoplasmas to diverse environments of their insect and plant hosts. J. Bacteriol. 188, 3682-3696. doi: 10.1128/JB.188.10.3682-3696.2006

Benson, D. A., Cavanaugh, M., Clark, K., Karsch-Mizrachi, I., Ostell, J., Pruitt, K. D., et al. (2018). GenBank. Nucleic Acids Res. 46, D41-D47.

Bertaccini, A., and Lee, I.-M. (2018). "Phytoplasmas: An update," in Phytoplasmas: Plant Pathogenic Bacteria - I: Characterisation and Epidemiology of Phytoplasma - Associated Diseases, eds G. P. Rao, A. Bertaccini, N. Fiore, and L. W. Liefting (Singapore: Springer).

Bertaccini, A., Duduk, B., Paltrinieri, S., and Contaldo, N. (2014). Phytoplasmas and phytoplasma diseases: a severe threat to agriculture. Am. J. Plant Sci. 5, 1763-1788. doi: 10.4236/ajps.2014.512191

Camacho, C., Coulouris, G., Avagyan, V., Ma, N., Papadopoulos, J., Bealer, K., et al. (2009). BLAST+: architecture and applications. BMC Bioinformatics 10:421. doi: $10.1186 / 1471-2105-10-421$

\section{SUPPLEMENTARY MATERIAL}

The Supplementary Material for this article can be found online at: https://www.frontiersin.org/articles/10.3389/fmicb. 2022.773608/full\#supplementary-material

Supplementary Figure 1 | Coverage pattern of raw read mapping results to the genome assembly. Rings from outside in: (1) Scale marks (kb). (2) Sequencing depth calculated from Illumina (dark blue; outward) and ONT (light blue; inward) raw reads mapped to the assembly using a sliding window of 200-bp. A filtering step was applied to include only the reads with high alignment scores. For Illumina reads, the cutoff was set at 200 , which identified 120,804 reads corresponding to an average sequencing depth of 47 -fold. For ONT reads, the cutoff was set at 1,000 , which identified 10,562 reads corresponding to an average sequencing depth of 79-fold. (3) Potential mobile unit (PMU) regions (orange). (4) Two duplicated chromosomal segments (green; positions: 316-391 and 391-467 kb).

Supplementary Figure 2 | Genome size estimation based on k-mer analysis. (A) A representative frequency distribution of $k$-mer depth $(k=21)$. The peak depth is 51. (B) Correspondence between genome size estimates and k-mer depths.

Supplementary Figure 3 | Protein sequence alignments of four characterized phytoplasma effectors. Individual effectors are identified using locus tags. Shaded background colors indicate the levels of sequence conservation. (A) SAP05. (B) SAP11/SWP1. (C) SAP54/PHYL1. (D) TENGU.

Supplementary Table 1 | List of potential mobile unit (PMU) core genes in the genome sequences analyzed.

Supplementary Table 2 | List of potential mobile unit (PMU) regions among the genome sequences analyzed. One strain, 'Ca. P. asteris' DY2014, was omitted because the PMU regions in this genome were reported to have complex structures and described in detail previously (Cho et al., 2019).

Supplementary Data 1 | Concatenated multiple sequence alignment of conserved single copy-genes used for inferring the molecular phylogeny shown in Figure 3. Simple text file in PHYLIP format.

Chang, S. H., Tan, C. M., Wu, C.-T., Lin, T.-H., Jiang, S.-Y., Liu, R.-C., et al. (2018). Alterations of plant architecture and phase transition by the phytoplasma virulence factor SAP11. J. Exp. Bot. 69, 5389-5401. doi: 10.1093/jxb/ery318

Chen, L.-L., Chung, W.-C., Lin, C.-P., and Kuo, C.-H. (2012). Comparative analysis of gene content evolution in phytoplasmas and mycoplasmas. PLoS One 7:e34407. doi: 10.1371/journal.pone.0034407

Cho, S.-T., Kung, H.-J., Huang, W., Hogenhout, S. A., and Kuo, C.-H. (2020a). Species boundaries and molecular markers for the classification of $16 \mathrm{SrI}$ phytoplasmas inferred by genome analysis. Front. Microbiol. 11:1531. doi: 10. 3389/fmicb.2020.01531

Cho, S.-T., Lin, C.-P., and Kuo, C.-H. (2019). Genomic characterization of the periwinkle leaf yellowing (PLY) phytoplasmas in Taiwan. Front. Microbiol. 10:2194. doi: 10.3389/fmicb.2019.02194

Cho, S.-T., Zwolińska, A., Huang, W., Wouters, R. H. M., Mugford, S. T., Hogenhout, S. A., et al. (2020b). Complete genome sequence of 'Candidatus Phytoplasma asteris' RP166, a plant pathogen associated with rapeseed phyllody disease in Poland. Microbiol. Resour. Announc. 9:e760-20. doi: 10.1128/MRA. 00760-20

Chung, W.-C., Chen, L.-L., Lo, W.-S., Lin, C.-P., and Kuo, C.-H. (2013). Comparative analysis of the peanut witches'-broom phytoplasma genome reveals horizontal transfer of potential mobile units and effectors. PLoS One 8:e62770. doi: 10.1371/journal.pone.0062770

Davis, R. E., Zhao, Y., Wei, W., Dally, E. L., and Lee, I.-M. (2017). 'Candidatus Phytoplasma luffae', a novel taxon associated with witches' broom disease of loofah, Luffa aegyptica Mill. Int. J. Syst. Evol. Microbiol. 67, 3127-3133. doi: 10.1099/ijsem.0.001980

Edgar, R. C. (2004). MUSCLE: multiple sequence alignment with high accuracy and high throughput. Nucleic Acids Res. 32, 1792-1797. doi: 10.1093/nar/gkh340

Felsenstein, J. (1989). PHYLIP - phylogeny inference package (version 3.2). Cladistics 5, 164-166. 
Gamboa, C., Cui, W., Quiroga, N., Fernández, C., Fiore, N., and Zamorano, A. (2019). Identification of 16SrIII-J phytoplasma effectors using a viral vector. Phytopathogenic Mollicutes 9:229. doi: 10.5958/2249-4677.2019.00115.4

Guindon, S., and Gascuel, O. (2003). A simple, fast, and accurate algorithm to estimate large phylogenies by maximum likelihood. Syst. Biol. 52, 696-704. doi: 10.1080/10635150390235520

Gundersen, D. E., and Lee, I.-M. (1996). Ultrasensitive detection of phytoplasmas by nested-PCR assays using two universal primer pairs. Phytopathol. Mediterr. 35, 144-151.

Guy, L., Roat Kultima, J., and Andersson, S. G. E. (2010). genoPlotR: comparative gene and genome visualization in R. Bioinformatics 26, 2334-2335. doi: 10.1093/ bioinformatics/btq413

Hogenhout, S. A., and Seruga Music, M. (2009). "Phytoplasma genomics, from sequencing to comparative and functional genomics - what have we learnt?," in Phytoplasmas: Genomes, Plant Hosts and Vectors, eds P. G. Weintraun and P. Jones (Wallingford: CABI), 19-36. doi: 10.1079/9781845935306.0019

Hogenhout, S. A., Oshima, K., Ammar, E.-D., Kakizawa, S., Kingdom, H. N., and Namba, S. (2008). Phytoplasmas: bacteria that manipulate plants and insects. Mol. Plant Pathol. 9, 403-423. doi: 10.1111/j.1364-3703.2008.00472.x

Hoshi, A., Oshima, K., Kakizawa, S., Ishii, Y., Ozeki, J., Hashimoto, M., et al. (2009). A unique virulence factor for proliferation and dwarfism in plants identified from a phytopathogenic bacterium. Proc. Natl. Acad. Sci. 106, 6416-6421. doi: 10.1073/pnas.0813038106

Huang, W., and Hogenhout, S. A. (2019). "Phytoplasma effectors have converged onto degrading plant transcription factors with fundamental roles in plant development and defense to insects," in Paper presentation at the IS-MPMI XVIII Congress Abstracts (Glasgow: IS-MPMI).

Huang, W., MacLean, A. M., Sugio, A., Maqbool, A., Busscher, M., Cho, S.-T., et al. (2021). Parasitic modulation of host development by ubiquitin-independent protein degradation. Cell 184, 5201-5214. doi: 10.1016/j.cell.2021.08.029

Hyatt, D., Chen, G.-L., LoCascio, P., Land, M., Larimer, F., and Hauser, L. (2010). Prodigal: prokaryotic gene recognition and translation initiation site identification. BMC Bioinformatics 11:119. doi: 10.1186/1471-2105-11-119

Jain, C., Rodriguez-R, L. M., Phillippy, A. M., Konstantinidis, K. T., and Aluru, S. (2018). High throughput ANI analysis of $90 \mathrm{~K}$ prokaryotic genomes reveals clear species boundaries. Nat. Commun. 9:5114.

Kanehisa, M., Goto, S., Furumichi, M., Tanabe, M., and Hirakawa, M. (2010). KEGG for representation and analysis of molecular networks involving diseases and drugs. Nucleic Acids Res. 38, D355-D360. doi: 10.1093/nar/gkp896

Kirdat, K., Tiwarekar, B., Thorat, V., Sathe, S., Shouche, Y., and Yadav, A. (2021). 'Candidatus Phytoplasma sacchari', a novel taxon - associated with Sugarcane Grassy Shoot (SCGS) disease. Int. J. Syst. Evol. Microbiol. 71:4591. doi: 10.1099/ ijsem.0.004591

Konstantinidis, K. T., and Tiedje, J. M. (2004). Trends between gene content and genome size in prokaryotic species with larger genomes. Proc. Natl. Acad. Sci. 101, 3160-3165. doi: 10.1073/pnas.0308653100

Krogh, A., Larsson, B., von Heijne, G., and Sonnhammer, E. L. L. (2001). Predicting transmembrane protein topology with a hidden Markov model: application to complete genomes. J. Mol. Biol. 305, 567-580. doi: 10.1006/jmbi.2000.4315

Krzywinski, M., Schein, J., Birol, İ, Connors, J., Gascoyne, R., Horsman, D., et al. (2009). Circos: an information aesthetic for comparative genomics. Genome Res. 19, 1639-1645. doi: 10.1101/gr.092759.109

$\mathrm{Ku}$, C., Lo, W.-S., and Kuo, C.-H. (2013). Horizontal transfer of potential mobile units in phytoplasmas. Mob. Genet. Elem. 3:e26145. doi: 10.4161/mge.26145

Kube, M., Mitrovic, J., Duduk, B., Rabus, R., and Seemüller, E. (2012). Current view on phytoplasma genomes and encoded metabolism. Sci. World J. 2012, 1-25. doi: 10.1100/2012/185942

Kube, M., Schneider, B., Kuhl, H., Dandekar, T., Heitmann, K., Migdoll, A., et al. (2008). The linear chromosome of the plant-pathogenic mycoplasma 'Candidatus Phytoplasma' mali. BMC Genomics 9:306. doi: 10.1186/1471-21649-306

Kuo, C.-H., and Ochman, H. (2009). Deletional bias across the three domains of life. Genome Biol. Evol. 1, 145-152. doi: 10.1093/gbe/evp016

Kuo, C.-H., Moran, N. A., and Ochman, H. (2009). The consequences of genetic drift for bacterial genome complexity. Genome Res. 19, 1450-1454. doi: 10.1101/ gr.091785.109
Kurtz, S., Phillippy, A., Delcher, A. L., Smoot, M., Shumway, M., Antonescu, C., et al. (2004). Versatile and open software for comparing large genomes. Genome Biol. 5:R12. doi: 10.1186/gb-2004-5-2-r12

Lagesen, K., Hallin, P., Rodland, E. A., Staerfeldt, H.-H., Rognes, T., and Ussery, D. W. (2007). RNAmmer: consistent and rapid annotation of ribosomal RNA genes. Nucleic Acids Res. 35, 3100-3108. doi: 10.1093/nar/gkm160

Lee, I.-M., Davis, R. E., and Gundersen-Rindal, D. E. (2000). Phytoplasma: phytopathogenic mollicutes. Annu. Rev. Microbiol. 54, 221-255.

Lee, I.-M., Gundersen-Rindal, D. E., Davis, R. E., and Bartoszyk, I. M. (1998). Revised classification scheme of phytoplasmas based on RFLP analyses of 16S rRNA and ribosomal protein gene sequences. Int. J. Syst. Evol. Microbiol. 48, 1153-1169. doi: 10.1099/00207713-48-4-1153

Lee, I.-M., Hammond, R., Davis, R., and Gundersen, D. (1993). Universal amplification and analysis of pathogen 16S rDNA for classification and identification of mycoplasmalike organisms. Phytopathology 83, 834-842. doi: 10.1094/phyto-83-834

Li, H. (2018). Minimap2: pairwise alignment for nucleotide sequences. Bioinformatics 34, 3094-3100. doi: 10.1093/bioinformatics/bty191

Li, H., and Durbin, R. (2009). Fast and accurate short read alignment with burrows-wheeler transform. Bioinformatics 25, 1754-1760. doi: 10.1093/ bioinformatics/btp324

Li, H., Handsaker, B., Wysoker, A., Fennell, T., Ruan, J., Homer, N., et al. (2009). The sequence alignment/map format and SAMtools. Bioinformatics 25, 20782079. doi: 10.1093/bioinformatics/btp352

Li, L., Stoeckert, C. J., and Roos, D. S. (2003). OrthoMCL: identification of ortholog groups for eukaryotic genomes. Genome Res. 13, 2178-2189. doi: 10.1101/gr. 1224503

Lo, W.-S., Gasparich, G. E., and Kuo, C.-H. (2018). Convergent evolution among ruminant-pathogenic Mycoplasma involved extensive gene content changes. Genome Biol. Evol. 10, 2130-2139. doi: 10.1093/gbe/evy172

Lo, W.-S., Huang, Y.-Y., and Kuo, C.-H. (2016). Winding paths to simplicity: genome evolution in facultative insect symbionts. FEMS Microbiol. Rev. 40, 855-874. doi: 10.1093/femsre/fuw028

Lowe, T., and Eddy, S. (1997). tRNAscan-SE: a program for improved detection of transfer RNA genes in genomic sequence. Nucleic Acids Res. 25, 955-964. doi: 10.1093/nar/25.5.955

Lu, M., An, H., and Li, L. (2016). Genome survey sequencing for the characterization of the genetic background of Rosa roxburghii Tratt and leaf ascorbate metabolism genes. PLoS One 11:e0147530. doi: 10.1371/journal.pone. 0147530

Lu, Y.-T., Li, M.-Y., Cheng, K.-T., Tan, C. M., Su, L.-W., Lin, W.-Y., et al. (2014). Transgenic plants that express the phytoplasma effector SAP11 show altered phosphate starvation and defense responses. Plant Physiol. 164, 1456-1469. doi: 10.1104/pp.113.229740

MacLean, A. M., Sugio, A., Makarova, O. V., Findlay, K. C., Grieve, V. M., Tóth, R., et al. (2011). Phytoplasma effector SAP54 induces indeterminate leaf-like flower development in Arabidopsis plants. Plant Physiol. 157, 831-841. doi: 10.1104/pp.111.181586

Maejima, K., Iwai, R., Himeno, M., Komatsu, K., Kitazawa, Y., Fujita, N., et al. (2014). Recognition of floral homeotic MADS domain transcription factors by a phytoplasmal effector, phyllogen, induces phyllody. Plant J. 78, 541-554. doi: $10.1111 /$ tpj.12495

Marçais, G., and Kingsford, C. (2011). A fast, lock-free approach for efficient parallel counting of occurrences of k-mers. Bioinformatics 27, 764-770. doi: 10.1093/bioinformatics/btr011

McCutcheon, J. P., and Moran, N. A. (2012). Extreme genome reduction in symbiotic bacteria. Nat. Rev. Microbiol. 10, 13-26. doi: 10.1038/nrmicro2670

Minato, N., Himeno, M., Hoshi, A., Maejima, K., Komatsu, K., Takebayashi, Y., et al. (2014). The phytoplasmal virulence factor TENGU causes plant sterility by downregulating of the jasmonic acid and auxin pathways. Sci. Rep. 4:7399. doi: 10.1038/srep07399

Mira, A., Ochman, H., and Moran, N. A. (2001). Deletional bias and the evolution of bacterial genomes. Trends Genet. 17, 589-596. doi: 10.1016/s0168-9525(01) 02447-7

Mittelberger, C., Stellmach, H., Hause, B., Kerschbamer, C., Schlink, K., Letschka, T., et al. (2019). A novel effector protein of apple proliferation phytoplasma 
disrupts cell integrity of Nicotiana spp. protoplasts. Int. J. Mol. Sci. 20:4613. doi: $10.3390 /$ ijms 20184613

Namba, S. (2019). Molecular and biological properties of phytoplasmas. Proc. Jpn. Acad. Ser. B 95, 401-418. doi: 10.2183/pjab.95.028

Nejat, N., Vadamalai, G., Davis, R. E., Harrison, N. A., Sijam, K., Dickinson, M., et al. (2013). 'Candidatus Phytoplasma malaysianum', a novel taxon associated with virescence and phyllody of Madagascar periwinkle (Catharanthus roseus). Int. J. Syst. Evol. Microbiol. 63, 540-548. doi: 10.1099/ijs.0.041467-0

Novichkov, P. S., Wolf, Y. I., Dubchak, I., and Koonin, E. V. (2009). Trends in prokaryotic evolution revealed by comparison of closely related bacterial and archaeal genomes. J. Bacteriol. 191, 65-73. doi: 10.1128/JB.01237-08

Ochman, H., and Davalos, L. M. (2006). The nature and dynamics of bacterial genomes. Science 311, 1730-1733. doi: 10.1126/science.1119966

Orlovskis, Z., and Hogenhout, S. A. (2016). A bacterial parasite effector mediates insect vector attraction in host plants independently of developmental changes. Front. Plant Sci. 7:885. doi: 10.3389/fpls.2016.00885

Orlovskis, Z., Canale, M. C., Haryono, M., Lopes, J. R. S., Kuo, C.-H., and Hogenhout, S. A. (2017). A few sequence polymorphisms among isolates of maize bushy stunt phytoplasma associate with organ proliferation symptoms of infected maize plants. Ann. Bot. 119, 869-884. doi: 10.1093/aob/mcw213

Oshima, K., Kakizawa, S., Nishigawa, H., Jung, H.-Y., Wei, W., Suzuki, S., et al. (2004). Reductive evolution suggested from the complete genome sequence of a plant-pathogenic phytoplasma. Nat. Genet. 36, 27-29. doi: 10.1038/ng1277

R Core Team (2019). R: a Language and Environment for Statistical Computing. Vienna: R Foundation for Statistical Computing

Robinson, J. T., Thorvaldsdottir, H., Winckler, W., Guttman, M., Lander, E. S., Getz, G., et al. (2011). Integrative genomics viewer. Nat. Biotechnol. 29, 24-26. doi: $10.1038 /$ nbt.1754

Sabater-Muñoz, B., Toft, C., Alvarez-Ponce, D., and Fares, M. A. (2017). Chance and necessity in the genome evolution of endosymbiotic bacteria of insects. ISME J. 11, 1291-1304. doi: 10.1038/ismej.2017.18

Saccardo, F., Martini, M., Palmano, S., Ermacora, P., Scortichini, M., Loi, N., et al. (2012). Genome drafts of four phytoplasma strains of the ribosomal group 16SrIII. Microbiology 158, 2805-2814. doi: 10.1099/mic.0.061432-0

Seruga Music, M., Samarzija, I., Hogenhout, S. A., Haryono, M., Cho, S.-T., and Kuo, C.-H. (2019). The genome of 'Candidatus Phytoplasma solani' strain SA-1 is highly dynamic and prone to adopting foreign sequences. Syst. Appl. Microbiol. 42, 117-127. doi: 10.1016/j.syapm.2018.10.008

Strohmayer, A., Moser, M., Si-Ammour, A., Krczal, G., and Boonrod, K. (2019). 'Candidatus Phytoplasma mali' genome encodes a protein that functions as an E3 ubiquitin ligase and could inhibit plant basal defense. Mol. Plant. Microbe Interact. 32, 1487-1495. doi: 10.1094/MPMI-04-19-0107- R

Sugawara, K., Honma, Y., Komatsu, K., Himeno, M., Oshima, K., and Namba, S. (2013). The alteration of plant morphology by small peptides released from the proteolytic processing of the bacterial peptide TENGU. Plant Physiol. 162, 2005-2014. doi: 10.1104/pp.113.218586

Sugio, A., and Hogenhout, S. A. (2012). The genome biology of phytoplasma: modulators of plants and insects. Curr. Opin. Microbiol. 15, 247-254. doi: 10.1016/j.mib.2012.04.002

Sugio, A., Kingdom, H. N., MacLean, A. M., Grieve, V. M., and Hogenhout, S. A. (2011a). Phytoplasma protein effector SAP11 enhances insect vector reproduction by manipulating plant development and defense hormone biosynthesis. Proc. Natl. Acad. Sci. 108, E1254-E1263. doi: 10.1073/pnas. 1105664108

Sugio, A., MacLean, A. M., Kingdom, H. N., Grieve, V. M., Manimekalai, R., and Hogenhout, S. A. (2011b). Diverse targets of phytoplasma effectors: from plant development to defense against insects. Annu. Rev. Phytopathol. 49, 175-195. doi: 10.1146/annurev-phyto-072910-095323

Tan, C. M., Lin, Y.-C., Li, J.-R., Chien, Y.-Y., Wang, C.-J., Chou, L., et al. (2021). Accelerating complete phytoplasma genome assembly by immunoprecipitation-based enrichment and MinION-based DNA sequencing for comparative analyses. Front. Microbiol. 12:766221. doi: 10.3389/fmicb.2021. 766221

Tatusov, R., Fedorova, N., Jackson, J., Jacobs, A., Kiryutin, B., Koonin, E., et al. (2003). The COG database: an updated version includes eukaryotes. BMC Bioinformatics 4:41. doi: 10.1186/1471-2105-4-41
The IRPCM Phytoplasma/Spiroplasma Working Team - Phytoplasma taxonomy group (2004). 'Candidatus Phytoplasma', a taxon for the wall-less, non-helical prokaryotes that colonize plant phloem and insects. Int. J. Syst. Evol. Microbiol. 54, 1243-1255. doi: 10.1099/ijs.0.02854-0

Tran-Nguyen, L. T. T., Kube, M., Schneider, B., Reinhardt, R., and Gibb, K. S. (2008). Comparative genome analysis of 'Candidatus Phytoplasma australiense' (subgroup tuf-Australia I; $r p-\mathrm{A}$ ) and 'Ca. Phytoplasma asteris' strains OY-M and AY-WB. J. Bacteriol. 190, 3979-3991. doi: 10.1128/JB.013 01-07

Wang, J., Song, L., Jiao, Q., Yang, S., Gao, R., Lu, X., et al. (2018a). Comparative genome analysis of jujube witches'-broom phytoplasma, an obligate pathogen that causes jujube witches'-broom disease. BMC Genomics 19:689. doi: 10.1186/ s12864-018-5075-1

Wang, N., Li, Y., Chen, W., Yang, H. Z., Zhang, P. H., and Wu, Y. F. (2018b). Identification of wheat blue dwarf phytoplasma effectors targeting plant proliferation and defence responses. Plant Pathol. 67, 603-609.

Wang, N., Yang, H., Yin, Z., Liu, W., Sun, L., and Wu, Y. (2018c). Phytoplasma effector SWP1 induces witches' broom symptom by destabilizing the TCP transcription factor BRANCHED1. Mol. Plant Pathol. 19, 2623-2634. doi: 10. 1111/mpp.12733

Waterhouse, A. M., Procter, J. B., Martin, D. M. A., Clamp, M., and Barton, G. J. (2009). Jalview version 2-a multiple sequence alignment editor and analysis workbench. Bioinformatics 25, 1189-1191. doi: 10.1093/bioinformatics/btp033

Weinert, L. A., and Welch, J. J. (2017). Why might bacterial pathogens have small genomes? Trends Ecol. Evol. 32, 936-947. doi: 10.1016/j.tree.2017.09.006

Wu, D., Hugenholtz, P., Mavromatis, K., Pukall, R., Dalin, E., Ivanova, N. N., et al. (2009). A phylogeny-driven genomic encyclopaedia of Bacteria and Archaea. Nature 462, 1056-1060. doi: 10.1038/nature08656

$\mathrm{Wu}, \mathrm{M}$., and Eisen, J. (2008). A simple, fast, and accurate method of phylogenomic inference. Genome Biol. 9:R151. doi: 10.1186/gb-2008-9-10-r151

Zerbino, D. R., and Birney, E. (2008). Velvet: algorithms for de novo short read assembly using de Bruijn graphs. Genome Res. 18, 821-829. doi: 10.1101/gr. 074492.107

Zhao, Y., and Davis, R. E. (2016). Criteria for phytoplasma 16Sr group/subgroup delineation and the need of a platform for proper registration of new groups and subgroups. Int. J. Syst. Evol. Microbiol. 66, 2121-2123. doi: 10.1099/ijsem.0. 000999

Zhao, Y., Wei, W., Davis, R. E., Lee, I.-M., and Bottner-Parker, K. D. (2021). The agent associated with blue dwarf disease in wheat represents a new phytoplasma taxon, 'Candidatus Phytoplasma tritici'. Int. J. Syst. Evol. Microbiol. 71:004604. doi: 10.1099/ijsem.0.004604

Zhao, Y., Wei, W., Lee, I.-M., Shao, J., Suo, X., and Davis, R. E. (2009). Construction of an interactive online phytoplasma classification tool, $i$ PhyClassifier, and its application in analysis of the peach X-disease phytoplasma group (16SrIII). Int. J. Syst. Evol. Microbiol. 59, 2582-2593. doi: 10.1099/ijs.0.010249-0

Zhou, J., Ma, F., Yao, Y., Deng, M., Chen, M., and Zhang, S. (2021). JWB phytoplasma effectors SJP1 and SJP2 induce lateral bud outgrowth by repressing the ZjBRC1-controlled auxin efflux channel. Plant Cell Environ. 44, 3257-3272. doi: $10.1111 /$ pce.14141

Conflict of Interest: The authors declare that the research was conducted in the absence of any commercial or financial relationships that could be construed as a potential conflict of interest.

Publisher's Note: All claims expressed in this article are solely those of the authors and do not necessarily represent those of their affiliated organizations, or those of the publisher, the editors and the reviewers. Any product that may be evaluated in this article, or claim that may be made by its manufacturer, is not guaranteed or endorsed by the publisher.

Copyright ( 2022 Huang, Cho, Lin, Tan, Chiu, Yang and Kuo. This is an open-access article distributed under the terms of the Creative Commons Attribution License (CC BY). The use, distribution or reproduction in other forums is permitted, provided the original author(s) and the copyright owner(s) are credited and that the original publication in this journal is cited, in accordance with accepted academic practice. No use, distribution or reproduction is permitted which does not comply with these terms. 